\title{
Incorporating simulated annealing algorithm in the Weibull distribution for valuation of investment return of Malaysian property development sector
}

\author{
Hamza Abubakar ${ }^{1,2,{ }^{*}}$ (D) and Shamsul Rijal Muhammad Sabri ${ }^{1}$ (D) \\ ${ }^{1}$ School of Mathematical Sciences, Universiti sains Malaysia, Penang, Malaysia \\ ${ }^{2}$ Department of Mathematics, Isa Kaita College of Education, Dutsin-Ma, Nigeria
}

Received: 6 June 2021 / Accepted: 28 September 2021

\begin{abstract}
In this study, a simulated annealing algorithm(SAA) has been incorporated in the Weibull Distribution (WD) for Valuation of Investment Return. The purpose is to examine the behaviour of investment's attractiveness in the Malaysian property development sector (MPDS) for a long-term investment period. The research intends is to produce parameters estimates of the WD using MIRR data extracted from the financial report of MPDS for 5 years investment period. The shape parameter of the WD reflects the effectiveness in maximizing the investment performance on MPDS with lower returns and is represented as the slope of the fitted line on a Weibull probability plot. The estimated results obtained using the Simulated annealing algorithm (SAA) has been compared with Differential Evolution (DE) and other existing estimation methods in terms of root mean square error (R-MSE) and coefficient of determination (R-Square). The findings revealed that Weibull distribution parameters estimated via Simulated annealing algorithm have good agreement with parameters estimated via Differential Evolution (DE) and other existing methods based on the transformed MIRR data from the MPDS. The study is expected to provide an overview of the investment behaviour for the long-term investment return in the MPDS. Therefore, SAA in estimating the WD parameters can serve as a good alternative approach for the assessment of the investment potential using MIRR data. The study will be extended to accommodate the growth rate arising from the financial data such as investment growth and insurance claim data.
\end{abstract}

Keywords: Investment modelling / modified internal rate of return / Weibull distribution / Maximum likelihood / simulated annealing algorithm / goodness-of-Fit tests

\section{Introduction}

In capital budgeting, many different criteria are used for forecasting and evaluating a project, measuring economic efficiency, and making appropriate investment decisions. Financial analysts research on examining the behaviour of investment adopt various matric measures such as the Return on Investment (ROI), Internal rate of return (IRR), Net Present Value (NPV), Return on Equity (ROE), return on asset (ROA) and Return On Investment (ROI). These messages are considered the most theoretically reliable tool since they correctly measure shareholder value creation [1]. Over the years, various models have been developed by various scholars to effectively explain investment behaviour and to forecast investment performance in different sectors of the economy based on their

\footnotetext{
* e-mail: zeeham4u2c@yahoo.com
}

ROI, NPV IRR, NPV, ROE ROA, ROI and MIRR. Modern portfolio theory pioneer by Harry Markowitz in 1952 is considered as one of the most popular techniques deployed. The theory showed how investors would make an entity with two investment instruments available optimally for investment and consumption decisions [2]. This theory offers a basis for constructing and selecting and examining portfolios based on predicted investment returns and the investor's risk tolerance [3]. Following the Modern portfolio theory by Markowitz, financial modelling has become necessary for any meaningful investment decision, as it plays a critical role in investment assessment and appropriate decision making [4-6].

There are many model frameworks developed by researchers in an attempt to examine or forecast the potential of investment return in a particular sector of the economy. A study in [7] proposed a modelling approach using interval mathematics and probability theory to deal with the underlying uncertainty associated with financial 
investment analysis and decision making. A modelling approach using a multiple time series was developed in [8], the based on a model framework in [9] by constructing a stochastic investment model for share dividend yields, price inflation, share dividends, and long-term interest rates. A model stock market investment model was developed based on a binary logit model associated with the financial market in [10]. A hybrid algorithm was developed based on the Moving Autoregressive Average (ARIMA) model fuzzy in [11]. A study on the decisionmaking for investment in Big data and its effects on supply chain coordination has been presented in [12]. A public housing construction project was analyzed based mathematical framework for investment projects in [13]. A machine learning-based investment in the insurance company was developed in [14]. Recently, an investment model framework based on the Partial Least SquareStructural equation model (PLS-SEM) was applied in examining the financial decision-making behaviour among real assets and financial investment [15]. Another recent mathematical model was developed based on economic principle for conducting factor financial analysis for industrial purposes in [16]. Apart from mathematical and statistical approaches applied in modelling and optimization of investment return, other novel approaches such as evolutionary and metaheuristic algorithms applied by various scholars include the work in [17] who applied genetic algorithms (GA) in portfolio optimization problems. A hybrid artificial bee colony (HABC) was applied in selecting an appropriate investment in [18]. A particle swarm optimization(PSO) was applied in the investment model for portfolio selection problems in financial engineering in [19]. Other variants of evolutionary computing known as hybrid metaheuristics algorithms were used in [20]. A simulated annealing algorithm (SA) with two machine learning techniques were applied in minimizing a business risk response in [21].

Various statistical distributions have been widely used for modelling different lifetime data such as reliability data set, wind speed data set and investment return data set. Weibull distribution is one of the few statistical models utilize due to their flexibility in fitting different applications of the analysis of varieties data set in different disciplines. This study utilized a Weibull distribution in fitting the modified internal rate of return data for investment analysis in MPDS. The suitability of Weibull distribution for reliability analysis for modelling distribution of survival or failure rate to describe real phenomena has been studied by various scholars. Investment analysis is one of many fields that employ many of the same statistical models for modelling the behaviour of prices and investment return. This is attributed to the fact that Weibull distribution provides the flexibility of data distribution useful for analysis in the emerging areas of research such as finance, stock prices and actuarial data in addition to its traditional engineering applications problem [22]. The optimal investment decision rule and asset equilibrium prices which depend on the assumed distribution of rates of return and empirical distributions vary with the assumed time interval (investment horizon) was proposed in [23]. The study measured the performance based on theoretical distributions for investment horizons ranging from one day to 4 years The post-modern portfolio based on a desired target return was proposed in [24-26].

This study employed the modified internal rate of return (MIRR) data generated from the MPDS financial report based on the assumption of Weibull distribution (WD). Although the Simulated annealing algorithm (SAA) has been applied in various optimization areas, it recorded tremendous achievement in parameters estimation of a linear and non-linear function. To the knowledge of the authors, Weibull distribution has not been used in investigating the behaviour of investment return. However, MIRR data has never been fitted based on the assumption of Weibull distribution (WD), therefore, this study is brandnew focusing on the stock investment modelling using the MIRR data of the MPDS. In this study, the Weibull distribution (WD) parameter has been estimated based on the MLE incorporated with Simulated annealing (SA) to investigate the behaviour of investment return in the MPDS. The contributions of the present study include;

- Propose an investment return modelling using Weibull distribution;

- Estimate a Weibull distribution parameters based on the maximum likelihood method (MLE) incorporated with the simulated annealing algorithm (SAA);

- Examine the behaviour of investment return of the Malaysian property development sector;

- Explore the effectiveness of the Simulated annealing algorithm (SAA) in estimating the parameters of Weibull distribution.

The effectiveness of the SAA in estimating the parameters of WD using the MIRR data set of MPDS will be explored based on goodness-of-Fit. The present study will be beneficial to investment decision-makers in making appropriate investment decisions with minimum risk and higher investment returns.

In Section 2 of this paper, we reported the materials and methods comprised of Modified Internal Rate of Return Modelling, Modified Internal Rate of Return on Weibull distribution, Simulated Annealing Algorithm (SAA). Following Section 2 is Section 3 which focused on Experimental Setup for Weibull distribution via Simulated Annealing Algorithm. The performances of Simulated annealing for Weibull parameter estimates were reported in Section 4 using Goodness-of-Fit statistical (GOF) based on MIRR data. Results and discussion were presented in Section 5. Section 6 concluded the studies and suggests further research studies.

\section{Modified internal rate of return modelling}

The investment strategy for holding the stock is by allocating a level amount of contribution $C$ at the beginning of the year for $K$ years. If we wish to hold the stock for the company chosen in the long term period, the stock valuation can be also seen by computing the MIRR [27]. At the same time, if the company declares dividends yearly, the cash dividends are reinvested and together deposited with the level contribution to enlarging the share units. At the end of $K$ years, we let all our share 
units earn the share capital which indicates the profit of our investment for $K$ years. If our share capital is less than our total contribution, we may expect our MIRR to be in a negative form. The detailed procedure of the investment return was documented in [27-29]. The Net Present Value (NPV) of stock investment is computed at time zero presented as follows,

$$
\begin{aligned}
N P V= & {\left[S_{K}^{(2)} P_{u_{K+1,2}}+B_{K}+\delta_{K}\right](1+r)^{-\frac{u_{K+1,1}-u_{1,1}}{365}} } \\
& -C \sum_{k=1}^{K+1} \mu_{k}(1+r)^{-\frac{u_{k+1,1}-u_{1,1}}{365}} \\
\equiv & F(K)(1+r)^{-\frac{u_{K+1,1}-u_{1,1}}{365}}-\sum_{k=1}^{K+1} C_{k}^{*}(1+r)^{-\frac{u_{k+1,1}-u_{1,1}}{365}}
\end{aligned}
$$

where $k=(1,2,3, \ldots, K)$ and $S_{k}^{(2)}$ is accumulated share unit after share issuance at the end of the year $k$ which can be computed as follow;

$$
S_{k}^{(2)}=\psi_{k} \times S_{k}^{(1)}
$$

where $\psi_{t}$ is the function of share issuance, $S_{k}^{(1)}$ is the share units at the beginning of the year $k$, and $F(K)$ is the terminal value investment fund to be let at the end of the year $K$ which can be computed as follows;

$$
F(K)=S_{K}^{(2)} P_{u_{k+1,2}}+B_{K}+\delta_{K}
$$

where $u_{K, 1}$ represent the date of share purchased and sold, $u_{k, 2}$ is the date of dividend and share issued based on the stock reported on year $k, P_{u_{k, 2}}$ defined the stock price at the date $u_{k, 2}, B_{k}$ represents the cash balance at the year $k, \delta_{K}$ defined as a cash dividend at year $k, r$ represents the modified IRR of the project development companies, $C$ is the yearly fixed contribution which can be computed as follows;

$$
C=\mu_{k} \frac{1}{C_{k}^{*}} .
$$

It is very important to choose the best potential stocks to hold in a holding term. Furthermore, holding a stock for a $K$-years period of the investment may vary in terms of MIRR. Some might choose the best point of time to start investing, but it is very difficult to identify it as the MIRR measure can only be observed yearly. Therefore, by assuming the MIRR for all starting times to invest are common, we may define the MIRR, denoted as $R_{t i K}$, as a random variable $(\mathrm{RV})$ with the mean $E\left(R_{t i K}\right)$ and variance and $\operatorname{Var}\left(R_{t i K}\right)$.

In an investment model framework, a positive value of profit may be obtained (or even be greater than our capital investment) as well as earning nothing. This indicates our capital of investment, $C$, could be infinite or even zero value. For some time $K$, our terminal investment $C\left(1+R_{K}\right)^{K}$ is in between 0 to infinity. Hence,

$$
0<C\left(1+R_{K}\right)^{K}<\rightarrow-1+R_{t i K}
$$

Since, $R_{t i K}>-1$, we define a non-negative transformed rate of return, $X_{t i K}$ such that

$$
X_{t i K}=1+R_{t i K}
$$

\section{Modified internal rate of return (MIRR) based on the weibull distribution}

This study considered investment returns for 62 companies from the Malaysian property development sector. The investment behaviour based on MIRR distribution from one (1) to eight (5) year investment periods have been considered (i.e., $K=1,2,3, \ldots, 5)$. The periods under study starts from 2014 and lasts until 2018, we set $t_{1}=2014$ and $t_{T}=2018$ hence, $T=5$. Furthermore, all companies' MIRR is counted in our study. For example, for a one-year investment period (i.e., $K=1$ ), we manage to obtain a maximum of $T=5$ multiplied by 62 companies understudy to obtain the sample size of 310 of MIRR data. The MIRR data has been transformed and assumed to follow the WD due to its flexibility in modelling different forms of data [30]. After acquiring the Weibull distribution parameters, the behaviour of investment return of the Malaysian property development sector for a long term investment period will be examined according to mean and variance.

In this study, the transformed MIRR data $\left(X_{i t K}\right)$ is assumed to come from the Weibull distribution i.e., $X_{t i K} \sim W b l r$ and $\left(\alpha_{K}, \beta_{K}, \eta_{K}\right)$. According to the observed MIRR data, the WD can be described based on PDF as follows.

\section{See equation (8) below.}

where $\alpha_{K}, \beta_{K}$ and $\eta_{K}$ are WD parameters. The mean of the WD in Equation (8) can be obtained as follows,

$$
E\left(X_{t i K}\right)=\eta_{K} \Gamma\left(1+\frac{1}{\beta_{K}}\right)+\alpha_{K}
$$

$$
f_{X_{t i K}}\left(\alpha_{K}, \beta_{K}, \eta_{K}\right)= \begin{cases}\frac{\beta_{K}}{\eta_{K}}\left(\frac{X_{t i K}-\alpha_{K}}{\eta_{K}}\right)^{\beta-1} e^{-\left(\frac{X_{t i K}-\alpha}{\eta_{K}}\right)^{\beta}}, & X_{t i K}>\alpha \\ 0, & X_{t i K} \leq \alpha\end{cases}
$$


where $\Gamma$ signifies a gamma function. The variance of the three parameters Weibull is deduced as follows.

$$
\operatorname{Var}\left(X_{t i K}\right)=\eta^{2}\left[\Gamma\left(1+\frac{2}{\beta_{K}}\right)-\Gamma^{2}\left(1+\frac{1}{\beta_{K}}\right)\right]
$$

a higher variance would generally provide lower profitability of companies at the same point in time. If we wish to re-write the MIRR based distribution in Equation (8), we may have the following,

$$
\begin{aligned}
& f_{R_{t i K}}\left(R_{t i K} ; \alpha_{K}, \beta_{K}, \eta_{K}\right) \\
& \quad=\frac{\beta_{K}}{\eta_{K}}\left(\frac{\left(R_{t i K}+1\right)-\alpha_{K}}{\eta_{K}}\right)^{\beta-1} e^{-\left(\frac{\left(R_{t i K}+1\right)-\alpha_{K}}{\eta_{K}}\right)^{\beta}}, R_{\tau i T}>-1
\end{aligned}
$$

This will also indicate that, mean and variance of Equation (11) can be re-written as in Equation (12) and (13) respectively as follows,

$$
\begin{gathered}
E\left(R_{t i K}\right)=\eta_{K} \Gamma\left(1+\frac{1}{\beta_{K}}\right)+\alpha_{K} \\
\operatorname{Var}\left(R_{t i K}\right)=\eta_{K}{ }^{2}\left[\Gamma\left(1+\frac{2}{\beta_{K}}\right)-\Gamma^{2}\left(1+\frac{1}{\beta_{K}}\right)\right]
\end{gathered}
$$

For a period of investment, $K$, the MLE of a random sample of $x_{1}, x_{2}, x_{3}, \ldots, x_{K^{*}}$ size $K^{*}$, where $K^{*} \in K$ has been considered. The likelihood function is presented as,

$L=\prod_{K=1}^{K *} \frac{\beta_{K}}{\eta_{K}}\left(\frac{\left(R_{t i K}+1\right)-\alpha_{K}}{\eta_{K}}\right)^{\beta_{K}-1} e^{-\left(\frac{\left(R_{t i K}+1\right)-\alpha_{K}}{\eta_{K}}\right)^{\beta_{K}}}, R_{t i K}>-1$

The log-likelihood function of Equation (14) is given as follows,

$$
\begin{aligned}
\operatorname{InL}= & \sum_{K=1}^{K *}\left[\operatorname{In}\left(\beta_{K}\right)+\left(\beta_{K}-1\right) \operatorname{In}\left(\left(r_{t i K}+1\right)-\alpha_{K}\right)\right. \\
& \left.-\beta_{K} \operatorname{In}\left(\eta_{K}\right)-\left(\frac{\left.\left(r_{t i K}+1\right)-\alpha_{K}\right)}{\eta_{K}}\right)^{\beta_{K}}\right]
\end{aligned}
$$

It is quite exhaustive to derive the gradient of the Weibull model in Equation (15) to attain the complicated objectives(fitness) function [31]. To address the complication and difficulties involved in the estimation of the parameters of WD, various metaheuristics algorithm (MA) architectures are used in estimating the resulting nonlinear objective functions [32-34]. MA are robust optimization and search procedure that only require computation of the likelihood (L) or log-likelihood (LL) function, but not its derivatives [35-37]. In this study, two parameters Weibull distribution is considered by setting the location parameter to zero $(\alpha=0)$, the resulting objectives (fitness) function has been evaluated via Simulated annealing algorithm (SA) adopted in [31]. The results obtained have been compared with the Deferential Evolution algorithm (DE) and other existing methods. The basic concept of a simulated annealing algorithm (SA) will be presented in the following section.

\section{Simulated annealing algorithm (SAA)}

Simulated annealing (SAA) is one of the first single-based stochastic metaheuristics optimization techniques inspired by the simulated thermodynamic process used in metallurgic for solidification studied in Statistical mechanics in which a material changes state while reducing its energy state to the lowest level (Kirkpatrick et al., 1983). This physical process occurs after the metal is removed from the heat source, when the molten material is physically rinsed, the temperatures are decreased very slowly as heat passes to the surrounding environment to crystallizes into one large crystalline lattice structure and metal becomes solid at this stage, the energy has reached its minimum level. In reaching the optimal solution, the SAA can be slow, because optimal results require a very slow lowering of the temperature with control from iteration to iteration (well organized and perfect structure). The resulting lattice structure is probably not ideal if the crystallization is too fast (imperfect structure). The benefits of SA over other metaheuristics include easy implementation, the feasibility of finding the global optimal solution even after finding a local optimal solution and satisfactory results are a guarantee with a relatively low number of iterations. The implementation of SA has been updated and extended to many mathematical and engineering domains. In this work, a robust and powerful heuristics search technique known as SA has been established for effective searching of the MLE fitness function for Weibull distribution (WD). $\mathrm{SA}$ is a stochastic approach designed to handle non-linear, complex and multi-dimensional global optimization problems objective function presented in minimization form as follows.

$$
f\left(\overrightarrow{\xi_{\text {opt }}}\right)=\min _{\overrightarrow{\xi_{i}} \in \vec{X}} f(\vec{\xi})
$$

Or in maximization form, as follows.

$$
f\left(\overrightarrow{\xi_{\text {opt }}}\right)=\max _{\overrightarrow{\xi_{i}} \in \vec{X}} f(\vec{\xi})
$$

where the variable $\xi_{i} \in \lambda$ is variable to be estimated via SA. Most optimization techniques use this interpretation. The basic steps of the SA algorithm have been presented as follows.

Step 1. Initialize - Start with a random initial placement $\tau_{i}$ according to Equation (18). Initialize a very high "temperature". $\tau_{0}$ (expected global minimum for the fitness function) and a feasible trial point $\xi^{(0)}$.

$$
\tau_{i}=\xi_{T}^{\min }+\tau_{1} *\left(\xi_{T}^{\max }-\xi_{T}^{\min }\right)
$$


where $\tau_{T}^{\min }$ and $\tau_{T}^{\max }$ defined as the initial and final temperature respectively. $N$ defined the number of temperatures, $\tau_{i} \in[1, N]$ has been chosen based on a specific cooling schedule considered as problem-dependent.

Step 2. Generate a new point $\xi^{(k)}$ randomly in a neighbourhood of the current point. If the point is infeasible, generate another random point until feasibility is satisfied (a variation of this step is explained later). Calculate the fitness function according to Equation (18) and Compute the difference according to Equation (19).

$$
\Delta f=f\left(\xi^{(k)}\right)-f\left(\xi^{(0)}\right)
$$

Step 3. Select the new best point. If $\Delta f<0$, in Equation (19) then take $\xi^{(k)}$ as the new best point $\xi^{(0)}$, set $f\left(\xi^{(k)}\right)=f\left(\xi^{(0)}\right)$ and go to Step 4. Otherwise, calculate the probability density function as follows.

$$
p(\Delta f)=\exp \left(\frac{\Delta f}{T_{K}}\right)
$$

Generate a random number $r$ according to Equation (21). If $\mathrm{r}<p(\Delta f)$, then take $\xi^{(k)}$ as the new best point $\xi^{(0)}$ and go to Step 4. Otherwise, go to Step 2.

$$
\xi^{(k)}= \begin{cases}\xi^{(k)}, & \text { if } \quad r \in[0,1]<p(\Delta f) \\ f\left(\xi^{(k)}\right)-f\left(\xi^{(0)}\right), & \text { otherwise }\end{cases}
$$

Step 4. If $k<L$, then set $k=k+1$ and go to Step 2. If $k>L$ and any of the stopping criteria is satisfied, then stop. Otherwise, go to Step 5.

Step 5. Set $K=K+1, k=1$; set $T_{K}=r T_{K-1}$; go to Step 2.

\section{Experimental setup for weibull parameter estimation via SAA}

Transformed Modified Internal rate of return (MIRR) data of the Malaysian property(MPDS) has been employed in this experiment. The main task of this program is to estimate the optimal parameters of the Weibull distribution that best maximizes the investment return of the property development (MPDS) for a long term investment period. Implementation of parameter searching method of Weibull distribution via Maximum likelihood was conducted by incorporating Simulated annealing algorithm. The source code for Weibull parameter estimates has been implemented on the $\mathrm{R}$ programming language. It was executed on a personal computer (PC) with Intel ${ }^{\circledR}$ Celeron ${ }^{\circledR}$ CPU T4800@ $8.4 \mathrm{GHz}$ processor with $8 \mathrm{~GB}$ RAM running on Windows 10. The R code, Microsoft Excel package and Mathematica used for this study are available from authors on request.

\section{Implementation of SAA on the estimation of WD parameters}

To estimate the parameters of the WD, we have to maximize $\mathrm{L}$ (or $\mathcal{L}$ ) using a simulated annealing approach based on the following steps;
Step 1. Generate an initial random solution $\xi_{0}$.

Step 2. Specify the values of the parameters $\beta, \eta$ and $\alpha$, say $\beta_{0}, \eta_{0}$ and $\alpha_{0}$ respectively.

Step 3. Specify the sample size of the MIRR distribution size $x_{i} \in\left[x_{1}, x_{2}, x_{3}, \ldots, x_{N}\right]$.

Step 4. Fit the MIRR data based on Weibull distribution $X_{t i K} \sim w b l r n d(\beta, \eta, \alpha)$.

Step 5. Select random to initialize the temperature $T_{0}>0$. Set the count $j=0$.

Step 6. Set the initial value of control parameters, temperature, Temp, cooling, Cool and V.

Step 7. Set $\xi=\xi^{*}=\xi_{0}$ and thus, compute $f(\xi)=f$ $\left(\xi^{*}\right)=f\left(\xi_{0}\right)=-L\left(\xi_{0} \mid x\right)$

Step 8. Initial value of Weibull parameters can be searched by generating up to $j$ sets of $\xi=\left(\alpha_{K}, \beta_{K}, \eta_{K}\right)$.

For $j=1,2, \ldots, J$, the best parameters, $\xi_{0}$ initially searched by SAA $\xi_{0}=\xi_{j}\left|\max _{j=1,2, \ldots, J}\right|\left[L\left(\alpha, \beta, \eta \mid X_{t i K}\right)\right]$.

Step 9. Based on step 4, estimate the parameters $\beta$, $\eta$ and $\alpha$ via the maximum likelihood method and Simulated an annealing algorithm in $\mathrm{R}$ packages.

Step 10. Compute the mean and the variance of the estimated values obtained from the $\mathrm{N}$ iterations for every parameter.

Step 11. Compute the RMSE and R-square statistic associated with each estimate of a vector of unknown parameters $\xi=(\beta, \eta, \alpha)=(\hat{\beta}, \hat{\eta}, \hat{\alpha})$.

Step 12. Repeat the entire process for the sizes $n_{T}$ at the number of iteration $N=10000$.

where, $L\left(\xi_{j} \mid X_{t i K}\right)$ is the log-likelihood function in Equation (15). The goal of SA is to estimate the value of the parameters that maximize the investment return in the MPDS over the investment period.

\section{Performance measurement}

Statistics are employed to determine how closely a particular distribution is for a given ECDF that matches the associated distribution for a particular dataset [38]. To examine whether a theoretical cumulative density function (CDF) is suitable to describe the transformed MIRR distributions data during the investment period or not, several tests are used for validating the accuracy of the predicted MIRR distribution obtained from the Weibull CDF. Finally, an optimal CDF is chosen for further evaluation of the investment return of the MPDS. The results for the goodness of the fitness test were computed using the Microsoft Excel package.

\section{Root mean square error (RMSE)}

In this study, RMSE is employed to assess the error accumulation of the fitted distribution model based on the Weibull distribution [39]. The RMSE equation is presented as follows,

$$
\mathrm{RMSE}=\sqrt{\frac{1}{n} \sum_{i=1}^{n}\left[\left(\hat{F}\left(X_{t i K}\right)-\bar{F}\right)^{2}\right]}
$$


where $n$ is defined as the number of intervals, $\hat{F}\left(X_{t i K}\right)$ is the actual fitted MIRR distribution data, $\bar{F}$ is the predicted MIRR data using the Weibull distribution. The GOF is considered a better one when the RMSE approaches zero.

\section{Coefficient of determination (R-Square)}

The coefficient of determination is the degree of correlation between the expected and observed values, which is a quantification of the estimated transformed MIRR PDF and the encountered PDF based on the Weibull distribution in the paper. The determination $R^{2}$ is described in [40] as follows,

$$
R^{2}=\frac{\sum_{i=1}^{n}\left[\left(\hat{F}\left(X_{t i K}\right)-\bar{F}\right)^{2}\right]}{\sum_{i=1}^{n}\left[\left(\hat{F}\left(X_{t i K}\right)-\bar{F}\right)^{2}\right]+\left[\sum_{i=1}^{n}\left(F_{n}\left(X_{t i K}\right)-\hat{F}\left(X_{t i K}\right)\right)^{2}\right]}
$$

where $n$ is defined as the number of intervals in which the MIRR is divided into, $\hat{F}\left(X_{t i K}\right)$ is the estimated cumulative distribution function of MIRR, $\hat{F}$ is the average frequency distribution of all the intervals given as follows.

$$
\bar{F}=\frac{1}{n} \sum_{i=1}^{n} \hat{F}\left(X_{t i K}\right)=\frac{1}{n} \times 1=\frac{1}{n}
$$

where a function $F_{n}\left(X_{t i K}\right)$ is the empirical distribution function of the MIRR data, given as follows.

$$
F_{n}\left(X_{t i K}\right)=\frac{1}{n} \sum_{i=1}^{n} g\left(x_{(i)} \leq x\right)
$$

where $g\left(x_{(i)} \leq x\right)$ is defined as follows.

$$
g\left(x_{(i)} \leq x\right)= \begin{cases}1, & \text { if } x_{(i)} \leq x \\ 0, & \text { otherwise }\end{cases}
$$

where $x_{(1)}, x_{(2)}, x_{(3)}, \ldots, x_{(n)}$ are observations in ascending order i.e., $x_{(1)}<x_{(2)}<x_{(3)}, \ldots,<x_{(n)}[41]$.

\section{Results and discussions}

The unknown parameters of the WD have been estimated via simulated annealing algorithm (SAA) and compared the results with the results estimated via Differential Evolution (DE), numerical methods (NM) and moments methods (MM). The statistical analysis in this study includes the estimated values of shapes and scales parameters, Log-likelihood value and GOF of MIRR data on WD for long term investment period ( 5 years). Based on the estimated parameters of the WD, the histogram of transformed MIRR data and the fitted Weibull Cumulative density function (WCDF) from 1 to 5 years investment period have been presented in Figure 1 until Figure 5

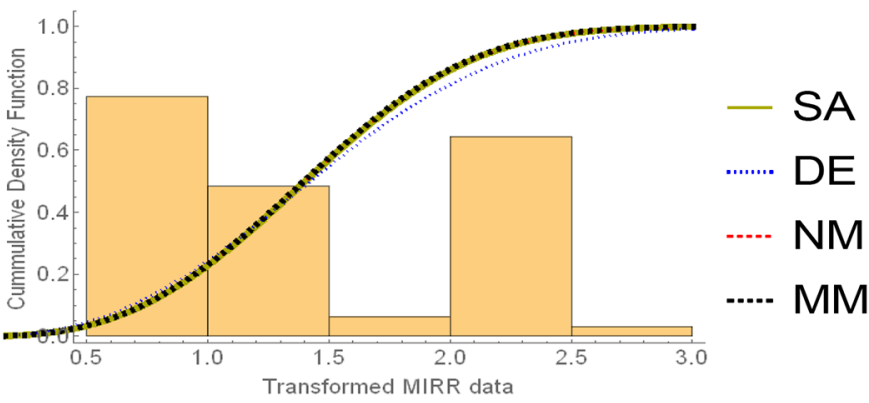

Fig. 1. Fitted CDF of transformed MIRR on Histogram for 1 year investment period.

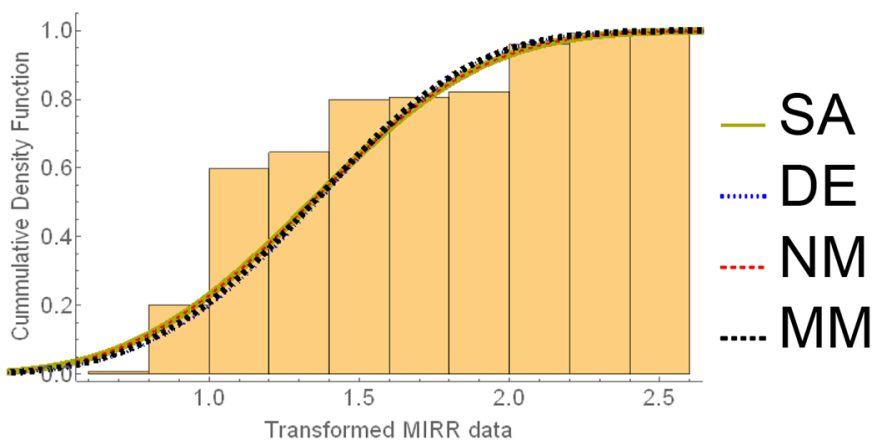

Fig. 2. Fitted CDF of transformed MIRR on Histogram a 2-year investment period.

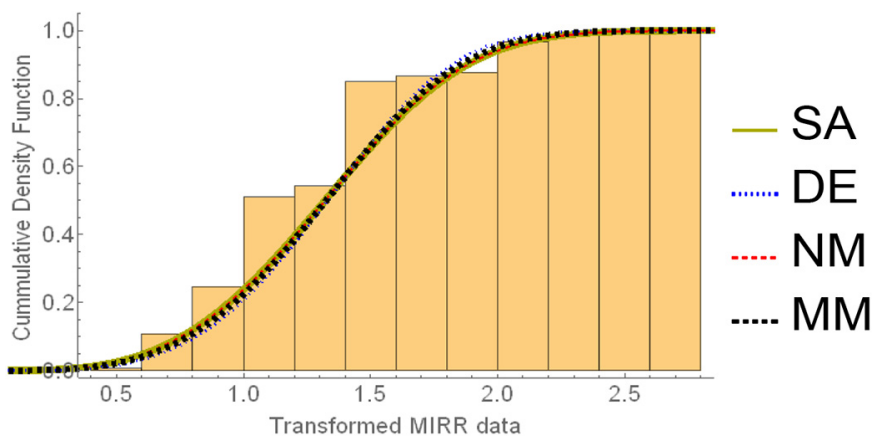

Fig. 3. Fitted CDF of transformed MIRR on Histogram for a 3 -year investment period.

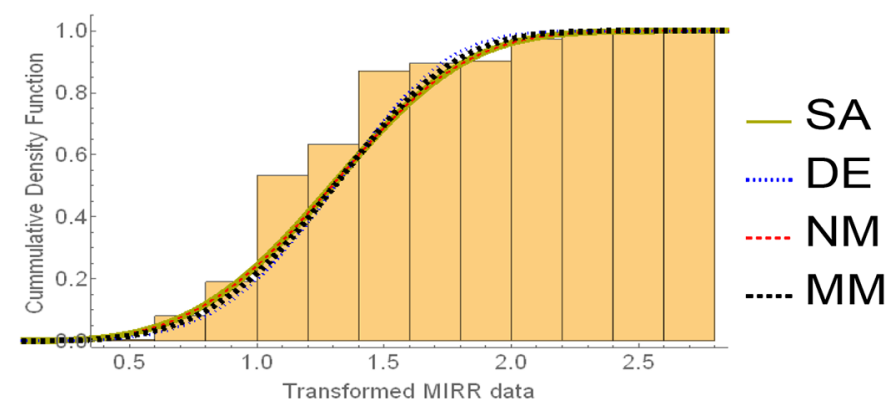

Fig. 4. Fitted CDF of transformed MIRR on Histogram for a 4-year investment period. 
respectively. The goodness of fitness statistical measures of performance have been displayed in Figures 6 and 7. It can be observed that there are virtually no differences between the estimates obtained by all the estimation methods. This revealed that SAA has good agreement with the existing methods. The graphs of the fitted Weibull models to the MIRR data revealed that the distribution is a poor fit for a short time investment period. On the other hand, the graphs for the WD appear to fit the MIRR data as the investment period increases with the increases in stock size. The results showed that the WD was a good fit for the long term investment period. The Weibull CDFs are a wellfitted distribution model for MIRR data for the Malaysian property development sector (MPDS) for the long-term investment period.

\section{Analysis of the findings}

The performance analysis of the investment return of the MPDS has been examined based on their MIRR data on the assumption of the Weibull distribution model. Figures 6 and 7 have displayed the performance of different estimation methods in terms of the RMSE and R -Square as the goodness-of-fit (GOF) statistics for the stock size from 62, up to 310 . The result of the GOF statistics revealed that estimation methods employed in this study performed well with the coefficient of determination much closer to 1 . On the other hand, the RMSE are closer to zero in all cases, which indicate that the estimation methods performed within the same limit. The results displayed in Figures 1, 2, 3, 4 and 5 have been supported by the lower RMSE and high R-square. This reveals that there are virtually no differences between the estimated values obtained via SAA, DE, NM and MM. This is not surprising because the objective function in all cases is the loglikelihood function. The difference in these methods being only in the way the objective function is maximized; one is through differentiation and the other by optimization. The numerical method (NM) and moment methods (MM) revealed better performance when stock size (SS) is small. The Estimates obtained are consistently better as the complexity increases, that is sample size increases. The main estimation theory states unequivocally that the larger the SS, the better the estimate [42]. However, as the SS grows or more parameters are involved, the fitness function (likelihood function) to optimize becomes more difficult. As a result, deciding on sample size (SS) is a matter of compromise. More accurate the maximum likelihood estimation and the accuracy will increase with the samples increase [43]. The results of the RMSE and R-square in Figures 6 and 7 confirmed this claimed. The Weibull model has been accepted in all cases considered by the methods of estimation used in computing the parameter values. A closer look at Figure 6 was the RMSE which revealed that MM and SA displayed the smallest errors. It will be difficult to observe the R-square behaviour for all the estimation methods in terms of better performance. However, it is quite clear that they display similar behaviour for all performance metrics used. This again indicates that the WD is a better fit for the MIRR data of

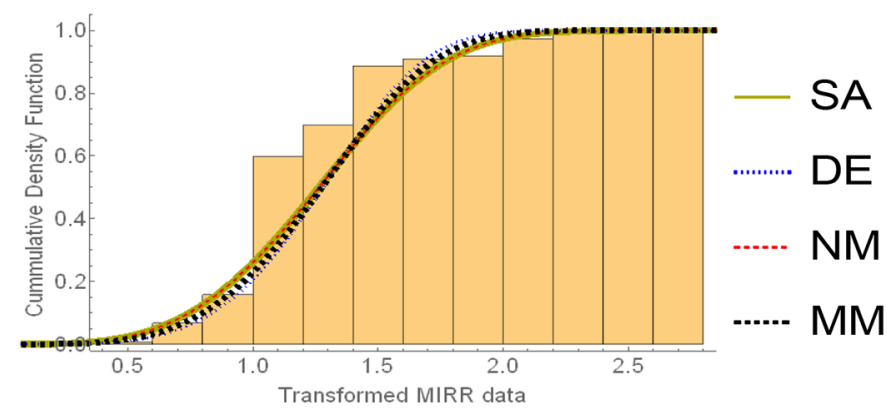

Fig. 5. Fitted CDF of transformed MIRR on Histogram for a 5-year investment period.

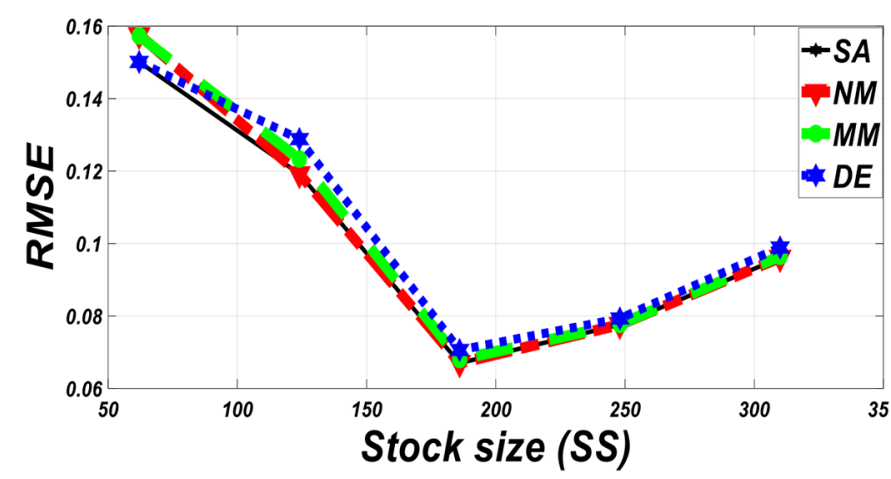

Fig. 6. Comparing RMSE of SA with other Weibull distribution estimation methods.

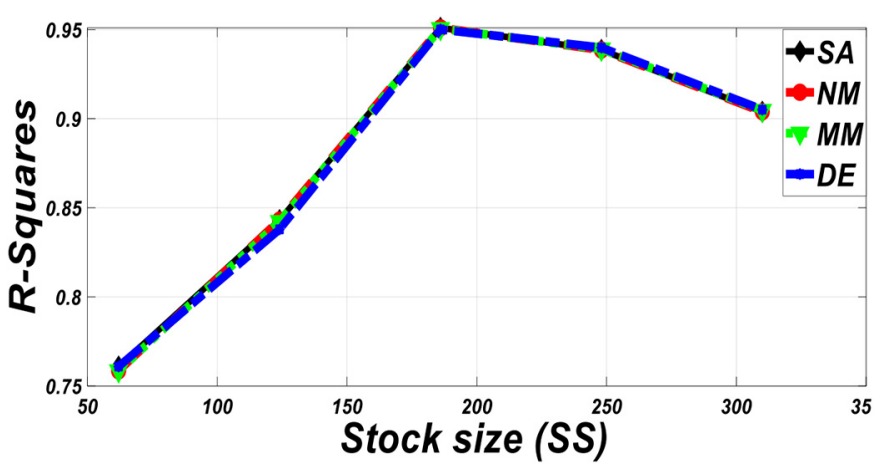

Fig. 7. Comparing R-Square of SA with other Weibull distribution estimation methods.

the MPDS. All estimation methods displayed similar trends based on the GOF to MIRR data.

Reports on the MIRR distribution analysis using the Weibull distribution produced objective performance measures. The Weibull analysis formulates the best applicable investment behaviour to reduce risk in the MPDS. Also, by using a probabilistic Weibull plot, it's possible to predict or forecast the behaviour of risk involved in the company and to plan a particular action to minimize the risk. If the MIRR data does describe a straight line it generally means, there is a steady rate of return in the investment. Now, if the slope of the fitted line is greater than one, it indicates an increase in a monotonic decrease in the investment return over time. The analysis considers the slope of the line (if straight) and gleans a few trends about 
investment behaviour. Detailed risk analysis reveals the impacts of a short time or long-time investment period, yet the longer the investment period, the less risk on the investment. This revealed that purchasing these companies shares may have a lower return for a short term period. This situation characterizes a risky investment. One of the objectives is to maximize the likelihood of the transformed MIRR. The longer the investment period, the high the return of the investment for this sector. To construct distributions, such an analysis can be generalized to other datasets. This was supported by the magnitudes of the RMSE and the R-square, which were very close. The Weibull distribution model gives an adequate representation of the MIRR data for the MPDS.

\section{Conclusions}

In this study, an investment modelling technique has been successfully presented on the assumption of Weibull distribution in fitting the modified internal rate of return. (MIRR) data of the MPDS. The data have allowed determining the distribution of MIRR from short time to long term investment period (1-5 years). The Weibull distribution parameters have been evaluated by a set of indicators defined from the MIRR distribution data. The capability of SA incorporated with the MLE in estimating two parameters Weibull distribution has been highlighted. The result obtained explored the effectiveness of the SAA in estimating the parameters of the Weibull distribution (WD) based on a real data set. We believe that using SA becomes more meaningful when the likelihood surface is more rugged (and has more dimensions) than the usual distributions. This study will be extended to three parameters Weibull distribution and extended Weibull distribution using a simulated annealing algorithm to investigate the investment behaviour of MPDS based on the transformed MIRR data for the long-term investment period. Furthermore, Weibull distribution will be modified and or extended to accommodate the growth rate arising from the financial data such as investment growth and insurance claim growth. Extension from our study, the different classes of statistical distribution can be adopted such as Gamma distribution, lognormal distribution, Tukey-Lambda Distribution, Rayleigh distribution etc to investigate the fit to MIRR data. These works are currently in progress and will be reported in a future article.

\section{References}

1. A. Marchioni, C.A. Magni, Investment decisions and sensitivity analysis: NPV-consistency of rates of return, Eur. J. Oper. Res. 268, 361-372 (2018)

2. H. Markowitz, Portfolio selection, J. Finance 7, 77-91 (1952)

3. H.M. Fabozzi, F.J. Gupta, F. Markowitz, The legacy of modern portfolio theory, J. Invest. 11, 7-22 (2002)

4. A.T. De Almeida-filho, D. Ferreira, D.L. Silva, Financial modelling with multiple criteria decision making: A systematic literature review, J. Oper. Res. Soc. 0, 1-19 (2020)

5. M. Brabazon, A. O'Neill, Biologically inspired algorithms for financial modelling, Springer Sci. Bus. Media. (2006)
6. A.G. Merikas, A.A. Merika, G. Koutroubousis, Modelling the investment decision of the entrepreneur in the tanker sector: Choosing between a second-hand vessel and a newly built one, 8839 (2010), doi: 10.1080/03088830802352053

7. A.K.M. Sherif Mohamed, Modelling project investment decisions under uncertaintyusing possibility theory, Int. J. Proj. Manag. 19, 231-241 (2001)

8. G.E. Tiao, G.C. Box, Modeling multiple time series with applications, J. Am. Stat. Assoc. 76, 802-816 (1981)

9. W.S. Chan, Stochastic investment modelling: a multiple time-series approach, Br. Actuar. J. 1, 545-591 (2002)

10. A.V. Rutkauskas, A. Miečinskiene, V. Stasytyte, Investment decisions modelling along sustainable development concept on financial markets, Technol. Econ. Dev. Econ. 14, 417-427 (2008)

11. N.M. Ralevi, The performance of the investment return prediction models: Theory and Evidence 44006 (September 2014), doi: 10.1109/SISY.2014.6923590

12. L.W. Aarssen, L. Crimi, Legacy, leisure and the 'work hardplay hard' hypothesis, The Open Psychology Journal, Need for structure: Adaptive and non-adaptive functions, Chinese J. Clin. Psychol. (2016)

13. L. Seliutina, M. Egorova, K. Bulgakova, Modelling of investment processes in the sphere of social house building, in: International Conference On Innovations In Science And Education, 2017, pp. 67-72

14. J.M. Sarabia, F. Prieto, V. Jordá, S. Sperlich, A note on combining machine learning with statistical modeling for financial data analysis 2005, 1-14 (2020), doi: 10.3390/ risks 8020032

15. Y. Hala, M.W. Abdullah, W. Andayani, G.B. Ilyas, M. Akob, The financial behavior of investment decision making between real and financial assets sectors, J. Asian Financ. Econ. Bus 7, 635-645 (2020)

16. A.G. Yankovoy, N.V. Melnik, Modified internal rate of return of the investment project, J. Appl. Manag. Investments 1, 502-508 (2012)

17. Z. Wang, R.T. Daigler, The performance of VIX option pricing models: Empirical evidence beyond simulation, J. Futur. Mark. (2011), doi: 10.1002/fut.20466

18. X. Wang, M. Zhang, J. Zhu, S. Geng, Spectral prediction of Phytophthora infestans infection on tomatoes using artificial neural network (ANN), Int. J. Remote Sens. (2008), doi: 10.1080/01431160701281007

19. Y. Hendrawan, D.F. Al Riza, Machine vision optimization using nature-inspired algorithms to model Sunagoke moss water status, Int. J. Adv. Sci. Eng. Inf. Technol. 6, 45-57 (2016)

20. I. Bavarsad Salehpoor, S. Molla-Alizadeh-Zavardehi, A constrained portfolio selection model at considering risk-adjusted measure by using hybrid meta-heuristic algorithms, Appl. Soft Comput. J. (2019), doi: 10.1016/j. asoc.2018.11.011

21. M.L. Erana-Diaz, M.A. Cruz-Chavez, R. Rivera-Lopez, B. Martinez-Bahena, E.Y. Avila-Melgar, M. Heriberto CruzRosales, Optimization for risk decision-making through simulated annealing, IEEE Access 8, 117063-117079 (2020)

22. M.Y.A. Christian, S. Victor, Comparison between the probability distribution of returns in the Heston model and empirical data for stock indexes, Phys. A Stat. Mech. Appl. 324, 303-310 (2003)

23. M. Muscettola, Probability of efficiency: Statistical implications that lead firms to achieve a minimal and sufficient 
'return-on-investment, J. Manag. Strateg. 5 (2014), doi: 10.5430/jms.v5n4p26.

24. P. Taleski, V. Bogdanovski, Statistical analyses of the performance of Macedonian investment and pension funds, Croat. Oper. Res. Rev. 6, 387-404 (2015)

25. J. Felton, P. Jain, True returns: Adjusting stock prices for cash dividends and stock splits, May, 192-205 (2018)

26. S. Sanni, B. O'Neill, Inventory optimisation in a threeparameter Weibull model under a prepayment system, Comput. Ind. Eng. 128, 298-304 (2019)

27. S. Rijal Muhammad Sabri, W. Mustafa Sarsour, Modelling on stock investment valuation for long-term strategy, J. Invest. Manag. 8, 60 (2019)

28. S. Kellison, stephen-kellison-theory-of-interest-3e.pdf, 2009

29. P. Protter, M. Capinski, T. Zastawniak, Mathematics for Finance: An Introduction to Financial Engineering, 111 (2004)

30. G.M. Thomas, Weibull parameter estimation using genetic algorithms and a heuristic approach to cut-set analysis, 1995, http://rave.ohiolink.edu/etdc/view?acc_num=ohiou1178901727

31. B. Abbasi, A.H. Eshragh Jahromi, J. Arkat, M. HosseinKouchack, Estimating the parameters of Weibull distribution using simulated annealing algorithm, Appl. Math. Comput. (2006), doi: 10.1016/j.amc.2006.05.063

32. A. Altin, A comparative study on optimization of machining parameters by turning aerospace materials according to Taguchi method, Int. J. Simul. Multidiscip. Des. Optim. (2017), doi: 10.1051/smdo/2016015

33. M.A. Khodja, M. Tadjine, M.S. Boucherit, M. Benzaoui, Tuning PID attitude stabilization of a quadrotor using particle swarm optimization (experimental), Int. J. Simul. Multidiscip. Des. Optim. (2017), doi: 10.1051/smdo/2017001

34. E.S. Maputi, R. Arora, Design optimization of a three-stage transmission using advanced optimization techniques, Int. J.
Simul. Multidiscip. Des. Optim. (2019), doi: 10.1051/smdo/ 2019009

35. S. Sathasivam, M.A. Mansor, M.S.M. Kasihmuddin, H. Abubakar, Election algorithm for random k satisfiability in the hopfield neural network, Processes (2020), doi: 10.3390/ pr8050568

36. H. Abubakar, M.L. Danrimi, Hopfield type of artificial neural network via election algorithm as heuristic search method for random boolean k satisfiability, Int. J. Comput. Digit. Syst. 10, 659-673 (2021)

37. H. Abubakar, S. Rijal, M. Sabri, S.A. Masanawa, S. Yusuf, Modified election algorithm in hopfield neural network for optimal random k satisfiability representation, Int. J. Simul. Multidisci. Des.Optim. 16, 1-13 (2020)

38. T. Sultana, F. Muhammad, M. Aslam, Estimation of parameters for the lifetime distributions 12, 77-92 (2019)

39. W. Wang, Y. Lu, Analysis of the Mean Absolute Error (MAE) and the Root Mean Square Error (RMSE) in assessing rounding model (2018), doi: 10.1088/1757-899X/ $324 / 1 / 012049$

40. I. Pobočíková, Z. Sedliačková, M. Michalková, Transmuted Weibull distribution and its applications, MATEC Web Conf. 157, 1-11 (2018)

41. H. Jiang, J. Wang, J. Wu, W. Geng, Comparison of numerical methods and metaheuristic optimization algorithms for estimating parameters for wind energy potential assessment in low wind regions, Renew. Sustain. Energy Rev. (2017), doi: 10.1016/j.rser.2016.11.241

42. A. Bensoussan, Estimation theory, 48 (2018)

43. K. Tashkova, J. Šilc, N. Atanasova, S. Džeroski, Parameter estimation in a nonlinear dynamic model of an aquatic ecosystem with meta-heuristic optimization, Ecol. Modell. (2012), doi: 10.1016/j.ecolmodel.2011.11.029

Cite this article as: Hamza Abubakar, Shamsul Rijal Muhammad Sabri, Incorporating simulated annealing algorithm in the Weibull distribution for valuation of investment return of Malaysian property development sector, Int. J. Simul. Multidisci. Des. Optim. 12, 22 (2021) 